\title{
Whole-exome sequencing identifies a novel mutation (R367G) in SCN5A to be associated with familial cardiac conduction disease
}

\author{
RONG YU ${ }^{1}$, XUE-FENG FAN ${ }^{2}$, CHAN CHEN $^{1}$ and ZHENG-HUA LIU ${ }^{3}$ \\ ${ }^{1}$ Department of Anesthesiology, The Second Xiangya Hospital of Central South University, Changsha, Hunan 410011; \\ ${ }^{2}$ Department of Orthopaedics, Xiangya Hospital, Central South University, Changsha, Hunan 410010; ${ }^{3}$ Department of \\ Thoracic Surgery, The Second Xiangya Hospital of Central South University, Changsha, Hunan 410011, P.R. China
}

Received April 16, 2016; Accepted March 16, 2017

DOI: $10.3892 / \mathrm{mmr} .2017 .6592$

\begin{abstract}
Cardiac conduction disease is a primary cause of sudden cardiac death. Sodium voltage-gated channel- $\alpha$ subunit 5 (SCN5A) mutations have been reported to underlie a variety of inherited arrhythmias. Numerous disease-causing mutations of SCN5A have been identified in patients with $\geq 10$ different conditions, including type 3 long-QT syndrome and Brugada syndrome. The present study investigated a family with a history of arrhythmia, with the proband having a history of arrhythmia and syncope. Whole-exome sequencing was applied in order to detect the disease-causing mutation in this family, and Sanger sequencing was used to confirm the co-segregation among the family members. A missense mutation (c.1099C>G/p.R367G) of SCN5A was identified in the family and was observed to be co-segregated in all affected members of the family. The missense mutation results in a substitution of glycine for arginine, which may affect sodium transmembrane transport. The present study provides an accurate genetic test which may be used in individuals who exhibit no clinical symptoms.
\end{abstract}

\section{Introduction}

Cardiac conduction disease is the major risk factor for sudden cardiac death (SCD), and therefore is the primary cause of mortality from SCD worldwide $(1,2)$. The principal characteristics of the disease are sudden arrhythmia and syncope (3). The human voltage-gated cardiac sodium channel serves a role in cardiac conduction, and mutations in sodium channel genes have been demonstrated to be associated with cardiac conduction disease (4), including sodium voltage-gated channel- $\alpha$ subunit 5 (SCN5A) (5), sodium voltage-gated

Correspondence to: Dr Zheng-Hua Liu, Department of Thoracic Surgery, The Second Xiangya Hospital of Central South University, 139 Ren Ming Road, Changsha, Hunan 410011, P.R. China

E-mail:911fanfan@sina.com

Key words: sodium voltage-gated channel- $\alpha$ subunit 5, arrhythmia, whole-exome sequencing, arrhythmia-associated gene filtering channel- $\beta$ subunit 4 (6) and sodium voltage-gated channel- $\beta$ subunit 1 (7).

The gene SCN5A encodes the pore-forming subunit of the human cardiac sodium channel NaV1.5 (8) and mutations in this gene have been reported to be associated with a variety of arrhythmogenic disorders, including type 3 long-QT syndrome (9), atrial fibrillation (10), dilated cardiomyopathy (11), Brugada syndrome (8) and certain complex overlapping disorders (12).

The present study investigated a clinically-characterized family with a history of syncope and arrhythmia. A clear autosomal-dominant inheritance of arrhythmia had been identified in the family. Using whole-exome sequencing (WES), in combination with arrhythmia-associated gene filtering, a novel missense mutation (c.1099C >G/p.R367G) was identified in SCN5A, which may underlie the pathogenesis of this type of familial arrhythmia.

\section{Materials and methods}

Patients and subjects. The protocol of the present study was approved by the Review Board of the Second Xiangya Hospital of Central South University (Changsha, China) and the study participants gave informed consent. A total of 14 members of the family (5 affected, 7 unaffected and 2 unknown) were enrolled in the present study. Blood was obtained from the affected probands and family members. Subjects underwent medical tests, including 12-lead electrocardiogram (ECG; Fig. 1) and ultrasonic cardiogram (UCG), and their hospital records were analyzed. A further 200 healthy people were also enrolled to exclude the single nucleotide polymorphism (13).

Whole-exome sequencing. Genomic DNA was extracted using the DNeasy Blood \& Tissue kit (Qiagen, Inc., Valencia, CA, USA). The Novogene Bioinformatics Institute (Beijing, China) performed the exome capture, high-throughput sequencing and common filtering. All of the exomes were captured using SureSelect Human All ExonV5 kits (Agilent Technologies, Inc., Santa Clara, CA, USA) and were sequenced using the HiSeq2000 platform (Illumina, Inc., San Diego, CA, USA). Filtering strategies were as described in a previous study (13). The effect of variants were predicted by polyphen 2 


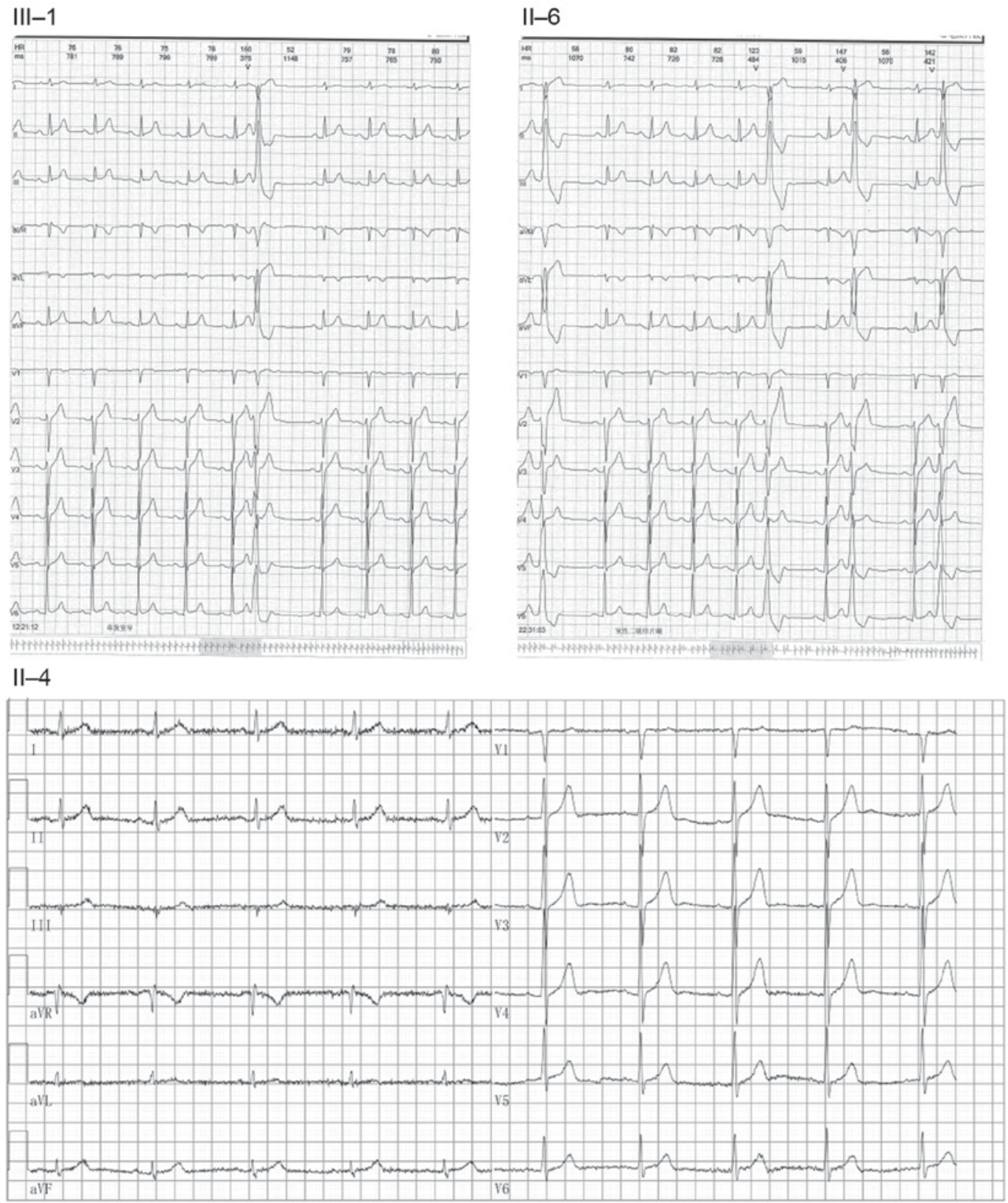

Figure 1. Clinical features of family members with cardiac conduction diseases. Electrocardiograms of the family members III-1, II-6 and II-4. Family members are identified by generations and numbers.

(http://genetics.bwh.harvard.edu/pph2/index.shtml), SIFT (http://sift.jcvi.org/) and MutationTaster (http://www.mutationtaster.org/).

Co-segregation analysis. Segregation analysis was applied in all family members according to the results of the WES. Primer pairs were designed using DNASTAR version 6.0 (Madison, WI, USA) and the sequences of primers are forward: CTACACCAGCTTCGATTCCTT; reverse: TGATCCCTT CTCCCTCAGAA.

\section{Results}

Clinical features. A Chinese family with a history of arrhythmia and syncope was identified (Fig. 2A). The proband, a 21-year-old college student from the Hunan province of central-southern China, had experienced syncope during strenuous physical exercise 6 months previously. A family history analysis demonstrated that the maternal uncle of the proband had experienced syncope 8 years previously and that the maternal grandmother of the proband had succumbed to an unknown cause during sleep. ECG and UCG analysis demonstrated that the maternal uncle of the proband exhibited sinus bradycardia. The proband and the mother of the proband exhibited ventricular premature beats (Fig. 1). The ECG of the sister of the proband was not obtained.

Genetic analysis. Data filtering excluded shared common variants present in the 1000 Genomes Project (http://www. internationalgenome.org/), dbSNP132 (https://www.ncbi. nlm.nih.gov/projects/SNP/) and ESP databases (http://evs. 

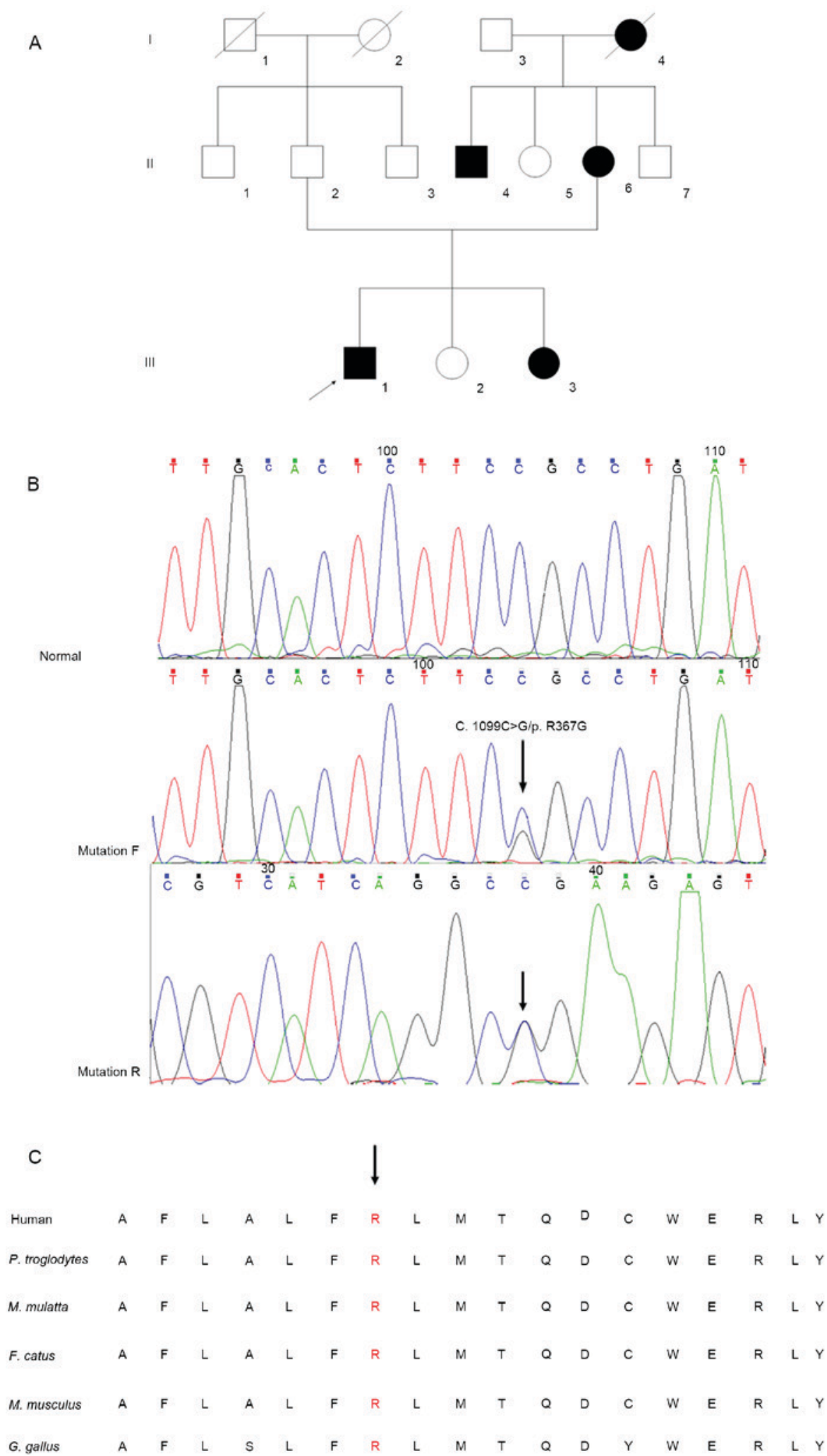

Figure 2. SCNA5 sequencing and alignment. (A) Pedigree of the family affected by arrhythmia. Family members are identified by generations and numbers. Squares, male family members; circles, female members; closed symbols, affected members; open symbols, unaffected members; arrow, proband. (B) Sequencing results of the SCN5A mutation. The sequence chromatogram indicates a C to G transition of nucleotide 1099. (C) Alignment of multiple SCN5A protein sequences across multiple species. The R367 affected amino acid is located in a highly-conserved amino acid region in different mammals Red indicates the R367 site. P. troglodytes, Pan troglodytes; M. mulatta, Macaca mulatta; F. catus, Felis catus; M. musculus, Mus musculus; G. gallus, Gallus gallus.

gs.washington.edu/EVS/). A total of 711 unique single-nucleotide polymorphisms were identified in the proband. Through screening of the variants of arrhythmia-associated genes, a novel missense mutation (p.R367G) of SCN5A was identified and Sanger DNA sequencing demonstrated that this mutation was co-segregated with affected members (Fig. 2B). The 
newly identified c.1099C $>$ G mutation in SCN5A was not observed in the 200 control individuals. The mutation was not present in the dbSNP and Exome Variant Server databases (evs.gs.washington.edu/EVS). Alignment of SCN5A amino acid sequences from human, Pan troglodytes, Mus musculus, Gallus gallus and other genomes (Fig. 2C) demonstrated that the affected amino acid was evolutionarily conserved. A total of three programs used for analyzing protein functions, polyphen2, SIFT and MutationTaster, predicted that the p.R367G variants of SCN5A may be damaging, deleterious and disease-causing, respectively.

\section{Discussion}

In the present study, a novel heterozygous mutation, c.1099C>G, was identified in the SCN5A gene, causing arrhythmia and syncope. Cosegregation analysis demonstrated the involvement of the mutation in the pathogenesis of the arrhythmic phenotype exhibited by the family in the present study. The diagnosis of arrhythmia in the proband was based on the ECG and UCG, which demonstrated a ventricular premature beat, as was additionally observed in the mother of the proband. The maternal uncle of the proband exhibited sinus bradycardia with a history of syncope and arrhythmia. In order to identify the disease-causing gene in the proband, WES analysis was used, in combination with arrhythmia-associated gene filtering, to explore the possible causative genes. A novel missense mutation (c.1099C $>$ G) in exon 9 of the SCN5A gene was identified, which resulted in an amino acid change from arginine to glycine at position 367 in the DI-S6 subunit of the NaV1.5 channel $(14,15)$. Bioinformatics analysis predicted that this mutation was disease-causing, and the site (R367) of SCN5A is evolutionarily conserved. The mutation was further investigated using co-segregation analysis, which demonstrated that all affected individuals in the present study carried the mutation, while the wild-type allele individuals did not. The results of the present study suggested that the SCN5A mutation led to the dysfunction of the NaV1.5 channel, which has been demonstrated to cause syncope during strenuous physical exercise (16).

The NaV1.5 channel $\alpha$ subunit consists of four homologous domains, and each domain contains six $\alpha$-helical transmembrane repeats (14). In the present study, the substituted amino acid p.R367G was located at the six $\alpha$-helical transmembrane segment of domain I-S6. The substitution of basic amino acid Arg by neutral amino acid Gly in position 367 of the SCN5A may affect transmembrane sodium transport (17). Numerous mutations in this site and region have been reported. For example, p.R367L and p.R367C were demonstrated to be associated with Brugada syndrome $(18,19)$. The mutation p.R367H was previously demonstrated to be involved in sudden unexplained nocturnal death syndrome (20). In addition, p.M369L, p.T370M and p.W374G were observed in Brugada syndrome and other arrhythmogenic disorders $(18,19,21)$. These previous results demonstrated that the site (R367) exerts an important function in the I-S6 domain of the NaV1.5 channel $\alpha$ subunit, and that this domain serves a role in the regulation of the gating properties of the channel.

Multiple lines of evidence support the notion that the mutation identified in the present study is associated with arrhythmia and syncope: i) This base was not identified in 200 control subjects, suggesting that it is not a common polymorphism; ii) no other meaningful mutations were identified in SCN5A or other genes; iii) this base change was not identified in other siblings who were not diagnosed with arrhythmia and syncope; iv) the mutation is evolutionally conserved in multiple animal species, suggesting that any substitution at this codon is not tolerated; and v) the same site mutations (p.R367H, p.R367L and p.R367C) have been previously identified to cause arrhythmia and SCD (18-20).

In conclusion, using whole-exome sequencing in combination with an arrhythmia-associated gene filtering method, a novel missense mutation (c.1099G >C/p.R367G) in SCN5A was identified to be a possible cause of a case of familial arrhythmia. The present study increases the understanding of SCN5A mutations, and contributes to potential genetic diagnosis and counseling of families with arrhythmia.

\section{Acknowledgements}

The authors of the present study would like to acknowledge the State Key Laboratory of Medical Genetics of China (Changsha, China), for technical assistance. The present study was supported by the Fundamental Research Funds for Central Universities of Central South University (grant no. 2016zzts163).

\section{References}

1. Smits JP, Veldkamp MW and Wilde AA: Mechanisms of inherited cardiac conduction disease. Europace 7: 122-137, 2005.

2. Kawaguchi T, Hayashi H, Miyamoto A, Yoshino T, Taniguchi A, Naiki N, Sugimoto Y, Ito M, Xue JQ, Murakami Y and Horie M: Prognostic implications of progressive cardiac conduction disease. Circ J 77: 60-67, 2013.

3. den Hoed M, Eijgelsheim M, Esko T, Brundel BJ, Peal DS, Evans DM, Nolte IM, Segrè AV, Holm H, Handsaker RE, et al: Identification of heart rate-associated loci and their effects on cardiac conduction and rhythm disorders. Nat Genet 45: 621-631, 2013.

4. Beltran-Alvarez P, Tarradas A, Chiva C, Pérez-Serra A, Batlle M, Pérez-Villa F, Schulte U, Sabidó E, Brugada R, Pagans S, et al: Identification of N-terminal protein acetylation and arginine methylation of the voltage-gated sodium channel in end-stage heart failure human heart. J Mol Cell Cardiol 76: 126-129, 2014.

5. Xiong Q, Cao L, Hu J, Marian AJ and Hong K: A rare loss-of-function SCN5A variant is associated with lidocaine-induced ventricular fibrillation. Pharmacogenomics J 14: 372-375, 2014.

6. Li RG, Wang Q, Xu YJ, Zhang M, Qu XK, Liu X, Fang WY and Yang YQ: Mutations of the SCN4B-encoded sodium channel $\beta 4$ subunit in familial atrial fibrillation. Int J Mol Med 32: 144-150, 2013.

7. Riuró H, Campuzano O, Arbelo E, Iglesias A, Batlle M, Pérez-Villa F, Brugada J, Pérez GJ, Scornik FS and Brugada R: A missense mutation in the sodium channel $\beta 1 \mathrm{~b}$ subunit reveals SCN1B as a susceptibility gene underlying long QT syndrome. Heart Rhythm 11: 1202-1209, 2014.

8. Bezzina CR, Barc J, Mizusawa Y, Remme CA, Gourraud JB, Simonet F, Verkerk AO, Schwartz PJ, Crotti L, Dagradi F, et al: Common variants at SCN5A-SCN10A and HEY2 are associated with Brugada syndrome, a rare disease with high risk of sudden cardiac death. Nat Genet 45: 1044-1049, 2013.

9. Blich M, Efrati E, Marai I, Suleiman M, Gepstein L and Boulous M: Novel Clinical Manifestation of the Known SCN5A D1790G Mutation. Cardiology 132: 228-232, 2015.

10. Ilkhanoff L, Arking DE, Lemaitre RN, Alonso A, Chen LY, Durda P, Hesselson SE, Kerr KF, Magnani JW, Marcus GM, et al: A common SCN5A variant is associated with PR interval and atrial fibrillation among African Americans. J Cardiovasc Electrophysiol 25: 1150-1157, 2014. 
11. Mann SA, Castro ML, Ohanian M, Guo G, Zodgekar P, Sheu A, Stockhammer K, Thompson T, Playford D, Subbiah R, et al: $\mathrm{R} 222 \mathrm{Q}$ SCN5A mutation is associated with reversible ventricular ectopy and dilated cardiomyopathy. J Am Coll Cardiol 60: 1566-1573, 2012.

12. Hothi SS, Ara F and Timperley J: p.Y1449C SCN5A mutation associated with overlap disorder comprising conduction disease, Brugada syndrome, and atrial flutter. J Cardiovasc Electrophysiol 26: 93-97, 2015.

13. Yu R, Liu L, Chen C and Shen JM: Exome Sequencing Identifies a Novel DES Mutation (R227C) in a Chinese Dilated Cardiomyopathy Family. Cardiology 137: 78-82, 2017.

14. Adsit GS, Vaidyanathan R, Galler CM, Kyle JW and Makielski JC: Channelopathies from mutations in the cardiac sodium channe protein complex. J Mol Cell Cardiol 61: 34-43, 2013.

15. Amin AS, Asghari-Roodsari A and Tan HL: Cardiac sodium channelopathies. Pflugers Arch 460: 223-237, 2010.

16. Shy D, Gillet L and Abriel H: Cardiac sodium channel NaV1.5 distribution in myocytes via interacting proteins: The multiple pool model. Biochim Biophys Acta 1833: 886-894, 2013.

17. van Hoorn F, Campian ME, Spijkerboer A, Blom MT, Planken RN van Rossum AC, de Bakker JM, Wilde AA, Groenink M and Tan HL: SCN5A mutations in Brugada syndrome are associated with increased cardiac dimensions and reduced contractility. PLoS One 7: e42037, 2012
18. Smits JP, Eckardt L, Probst V, Bezzina CR, Schott JJ, Remme CA, Haverkamp W, Breithardt G, Escande D, Schulze-Bahr E, et al: Genotype-phenotype relationship in Brugada syndrome: Electrocardiographic features differentiate SCN5A-related patients from non-SCN5A-related patients. J Am Coll Cardiol 40: 350-356, 2002.

19. Kapplinger JD, Tester DJ, Alders M, Benito B, Berthet M, Brugada J, Brugada P, Fressart V, Guerchicoff A, Harris-Kerr C, et al: An international compendium of mutations in the SCN5A-encoded cardiac sodium channel in patients referred for Brugada syndrome genetic testing. Heart Rhythm 7: 33-46, 2010.

20. Vatta M, Dumaine R, Varghese G, Richard TA, Shimizu W, Aihara N, Nademanee K, Brugada R, Brugada J, Veerakul G, et al: Genetic and biophysical basis of sudden unexplained nocturnal death syndrome (SUNDS), a disease allelic to Brugada syndrome. Hum Mol Genet 11: 337-345, 2002.

21. Hofman-Bang J, Behr ER, Hedley P, Tfelt-Hansen J, Kanters JK, Haunsøe S, McKenna WJ and Christiansen M: High-efficiency multiplex capillary electrophoresis single strand conformation polymorphism (multi-CE-SSCP) mutation screening of SCN5A: A rapid genetic approach to cardiac arrhythmia. Clin Genet 69: 504-511, 2006. 